\title{
Laparoscopic hepatectomy versus radiofrequency ablation for hepatocellular carcinoma: a systematic review and meta-analysis
}

This article was published in the following Dove Press journal:

Cancer Management and Research

\begin{abstract}
Xiaocheng $\mathrm{Li}^{1}$
Yu-Shen $\mathrm{Wu}^{2}$

Duke Chen ${ }^{3}$

Huapeng $\operatorname{Lin}^{4}$

'Department of Hepatobiliary Surgery, The First Affiliated Hospital of Hunan University of Medicine, Huaihua, Hunan, People's Republic of China; ${ }^{2}$ Chongqing Key Laboratory of Molecular Oncology and Epigenetics, The First Affiliated Hospital of Chongqing Medical University, Chongqing, People's Republic of China; ${ }^{3}$ Department of Oncology, The First Affiliated Hospital of Chongqing Medical University, Chongqing, People's Republic of China; ${ }^{4}$ Department of Intensive Care Unit, Affiliated Hangzhou First People's Hospital, Zhejiang University School of Medicine, Hangzhou, Zhejiang, People's Republic of China
\end{abstract}

Correspondence: Huapeng Lin Department of Intensive Care Unit, Affiliated Hangzhou First People's Hospital, Zhejiang University School of Medicine, 26I Huansha Road, Hang Zhou, Zhejiang 310006, People's Republic of China

Tel +860 2363693626

Fax +8602363693533

Email linhuapeng199I@I63.com
Aim: To compare the effectiveness of laparoscopic hepatectomy (LH) with that of radiofrequency ablation (RFA) for hepatocellular carcinoma (HCC).

Methods: We conducted a literature search without a language restriction to identify relevant available articles that had been published with the EMBASE and PubMed databases and the Cochrane Library. Studies comparing the outcomes of LH versus RFA for HCC were eligible for inclusion.

Results: A total of 10 studies with 1570 patients was included in this meta-analysis. The pooled results revealed that LH was superior to RFA in terms of the 5-year overall survival rate $(\mathrm{OR}=0.53,95 \% \mathrm{CI}=0.40,0.69, p<0.001)$. In the subgroup analysis of small HCCs, there was still a significantly better 5-year overall survival rate in the LH group compared with the RFA group ( $\mathrm{OR}=0.47,95 \% \mathrm{CI}=0.33,0.66, p<0.001)$. Additionally, the $\mathrm{LH}$ group had better 1 - and 3-year disease-free survival rate and a lower local recurrence rate, compared with the RFA group. However, the complication rate was higher in the LH group than the RFA group $(\mathrm{OR}=0.64,95 \% \mathrm{CI}=0.46,0.89, p=0.008)$.

Conclusion: Patients who underwent LH had a better long-term prognosis and a lower recurrence rate than those who received RFA. However, we did not obtain conclusive evidence for the superiority of LH over RFA for the treatment of HCCs due to the inclusion of retrospective studies in the present meta-analysis, and well-designed RCTs are needed.

Keywords: laparoscopic hepatectomy, radiofrequency ablation, hepatocellular carcinoma, overall survival, disease-free survival

\section{Introduction}

Hepatocellular carcinoma (HCC), which ranks as the fifth most common malignancy, is the second leading cause of cancer death worldwide due to its highly malignant nature. ${ }^{1}$ Several modalities of treatment, including liver transplantation, hepatic resection, and ablative therapies, have been recommended for use with curative intention for HCC.,3 Liver transplantation (LT), which simultaneously cures the tumor and the underlying cirrhosis, is recognized as the best choice for patients who met the Milan criteria. ${ }^{4}$ However, the shortage of donor organs, difficulty of the transplant operation, and substantial postoperative complications limit the application of LT.

Hepatic resection remains the mainstay of curative treatment for $\mathrm{HCC}{ }^{5}$ However, the high incidence of complications and the loss of liver function make resection not ideal for $\mathrm{HCC}$, especially for HCC combined with severe cirrhosis. Therefore, radiofrequency 
ablation (RFA), which is characterized by its ease of use and invasiveness, has recently been proposed as an alternative for resectable small HCC. Several studies and meta-analyses have shown that RFA has similar long-term outcomes but requires a shorter hospital stay and has fewer complications compared to those of open liver resection. Therefore, both RFA and hepatectomy have been recommended for the treatment of early stage HCC in the Barcelona Clinic Liver Cancer (BCLC) staging system. ${ }^{6}$

With the recent advances of laparoscopic technology, laparoscopic hepatectomy (LH) has been gradually adopted for the treatment of HCC, particularly small HCC. LH is believed to bear the advantages of RFA (minimally invasive and safe), while it has the features of open resection (complete removal of tumors, thus reducing the recurrence rate). ${ }^{7}$ Hence, several studies focused on the comparison between the LH and RFA for HCC, and came to conflict conclusions. The study by Casaccia et al revealed that LH was superior to RFA in terms of overall survival; nevertheless, the disease-free survival and complication rate were similar. ${ }^{8}$ Song et al found that the RFA group had a lower complication rate and that the LH group had a better disease-free survival, and there was no significant difference for overall survival. ${ }^{9}$ In a recent study by Yamashita et al, RFA was shown to have a comparable survival outcome but a lower morbidity compared with those of LH. ${ }^{10}$ Therefore, we performed the present metaanalysis to pool the results of the existing studies and to compare the efficacy and safety of RFA and LH for the treatment of HCC.

\section{Materials and methods}

\section{Search strategy}

We conducted a literature search without a language restriction to identify relevant available articles that had been published with the EMBASE, PubMed and the Cochrane Library databases from their inception to July 2018. The search terms included "laparoscopic resection", "laparoscopic hepatectomy", "laparoscopy", "minimally invasive surgical procedures", "radiofrequency ablation", and "hepatocellular carcinoma". We also reviewed the reference lists of the included studies for undetected relevant studies.

\section{Inclusion and exclusion criteria}

The inclusion criteria were as follows: (1) original research from nonrandomized controlled trials or randomized controlled trials (RCTs) among adults; (2) the interventions of interest were laparoscopic hepatectomy (including laparoscopic or laparoscopic-assisted liver resection or robotic-assisted liver resection) and radiofrequency ablation (including laparoscopic or percutaneous RFA); (3) the participants of interest were patients who were diagnosed with primary hepatocellular carcinoma; (4) the primary outcomes of interest were the overall and disease-free survival rate, and the secondary outcome of interest was the complication rate; (5) the odds ratio (OR) with the $95 \%$ confidence interval (CI) of the risk of the overall and disease-free survival rate were either provided or could be calculated; and (6) the most recent and complete study was included if data from the same population had been published more than once. The exclusion criteria were as follows: (1) participants were animals, children, and pregnant women; (2) patients whose permanent pathology after the procedure suggested benign focal nodular hyperplasia, inflammatory nodules, metastatic liver cancer, or primary liver cancer of other pathological types (such as cholangiocarcinoma); (3) the absence of data regarding the primary and secondary outcomes; and (4) the publication type was a case report, editorial, or a review.

Two investigators searched and independently reviewed all identified studies. If the two investigators were not able to reach a consensus about the eligibility of an article, the dispute was resolved through discussions with a third reviewer.

\section{Data extraction and quality assessment}

The following data were independently extracted from each study by two investigators: the first author's name, publication year, country where the study was performed, study design, and the baseline characteristics of the involved patients. The Jadad scale was used to assess the quality of RCTs. The qualitative assessment of observational studies was based on the Newcastle-Ottawa Scale (NOS), which assessed the included studies based on the population selection, study comparability, and outcome reporting. Each study was awarded a score from one to nine points.

\section{Statistical analysis}

The meta-analysis was conducted using the RevMan software version 5.3 (The Nordic Cochrane Center, Cochrane Collaboration, and Copenhagen, Denmark). The odds ratio with the $95 \%$ confidence interval was calculated to compare the overall and disease-free survival rates between the LH and RFA groups. For studies that did not provide the 
overall and disease-free survival rate, the survival data were extracted from the Kaplan-Meier curves by the Engauge Digitizer v.4.1 software. Heterogeneity among the included studies was qualitatively evaluated using a $\chi 2$-based Q test. A $p$-value of less than 0.05 showed that there was significant heterogeneity across the studies. The level of the heterogeneity between studies was evaluated with $\mathrm{I}^{2}$ statistics. $\mathrm{I}^{2}<50 \%$ was considered to demonstrate low or moderate heterogeneity, and a fixed- or random-effects model was applied; $\mathrm{I}^{2} \geq 50 \%$ demonstrated high heterogeneity, and a random-effects model was applied. The search strategy and statistical analysis methods were similar to those of our previous study. ${ }^{11}$

\section{Sensitivity analysis and assessment of the risk of bias}

A sensitivity analysis was performed by sequentially excluding one study and then recalculating the combined results of the remaining studies to assess whether the results had been markedly affected by the studies with a small sample size. The assessment of the risk of bias was studied with funnel plot analysis.

\section{Results}

\section{Search results and study characteristics}

A total of 2723 studies were screened out by searching the electronic databases and by manually searching the relevant reference lists, and 629 overlapping articles were identified. After the duplicates were identified and excluded, 2094 articles remained. We excluded unrelated reviews, case reports, systematic reviews, and meta-analyses based on the title or abstract, leaving 420 articles. Finally, the fulltext screening led to the inclusion of 10 studies with 1570 patients (553 for LH and 1017 for RFA) in this metaanalysis. The detailed steps of our literature search are shown in Figure 1. There were no RCTs that compared
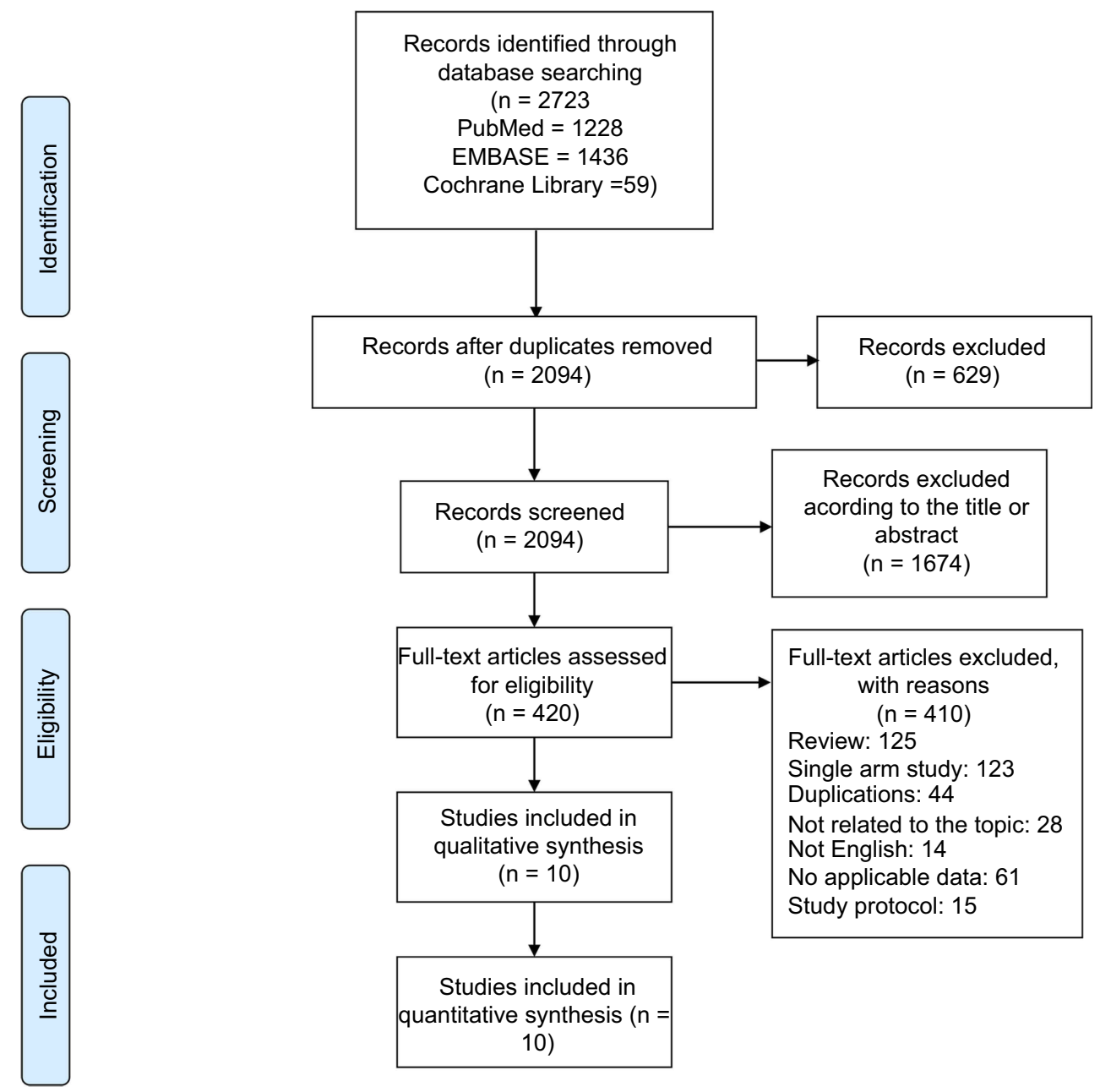

Figure I Flow chart of study selection. 


\begin{tabular}{|c|c|c|c|c|c|c|}
\hline 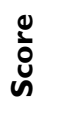 & $\infty$ & n & n & $\wedge \infty \infty$ & & \\
\hline 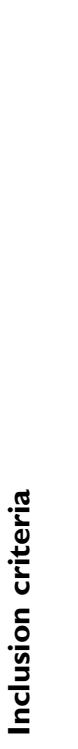 & 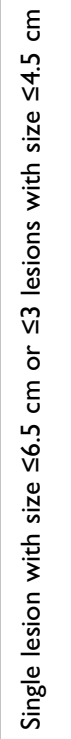 & \multicolumn{2}{|c|}{ 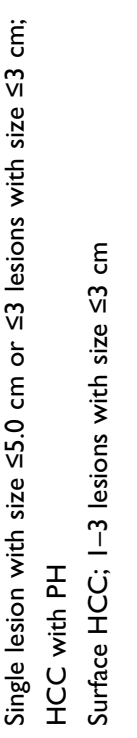 } & \multicolumn{3}{|c|}{ 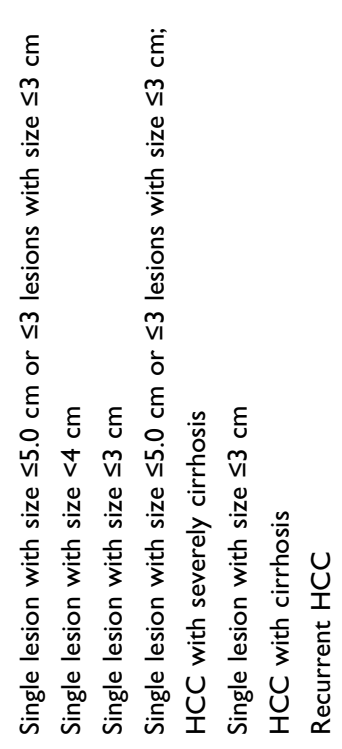 } \\
\hline 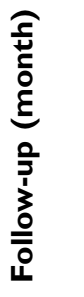 & \multicolumn{2}{|c|}{ 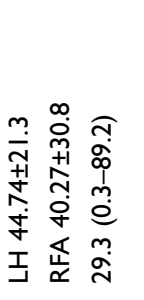 } & \multicolumn{4}{|c|}{ 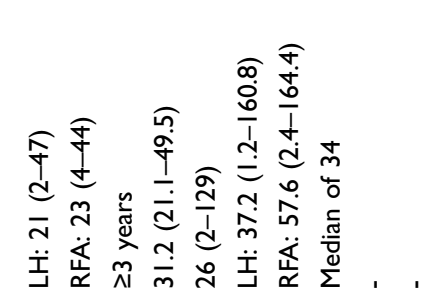 } \\
\hline 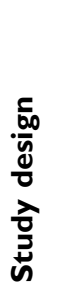 & 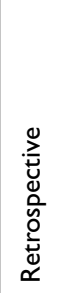 & 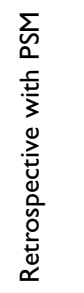 & 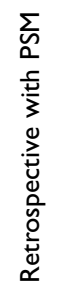 & 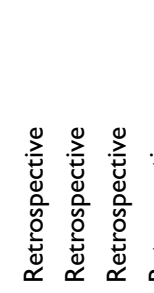 & 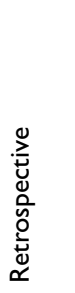 & 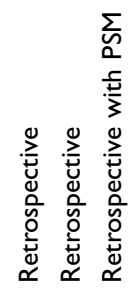 \\
\hline 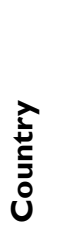 & 초 & 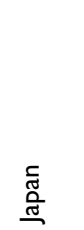 & 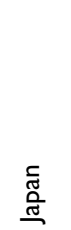 & 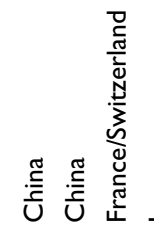 & 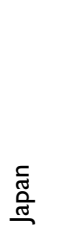 & 压 \\
\hline 弚 & 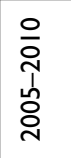 & 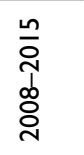 & $\frac{m}{\bar{c}}$ & 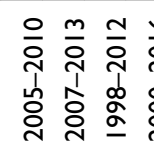 & 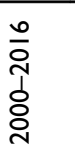 & 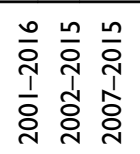 \\
\hline 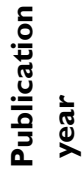 & $\begin{array}{l}\overline{\mathrm{N}} \\
\frac{1}{2} \\
\frac{\mathrm{n}}{2}\end{array}$ & $\frac{\circ}{2}$ & $\frac{0}{2}$ & 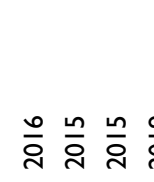 & $\frac{a}{\bar{n}}$ & 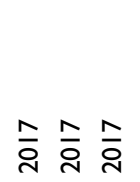 \\
\hline 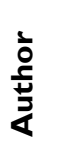 & 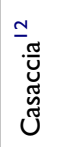 & $\begin{array}{l}\frac{m}{\pi} \\
\frac{\pi}{0} \\
\frac{\pi}{\pi} \\
\frac{\pi}{I}\end{array}$ & $\stackrel{ \pm}{\underline{o}}$ & 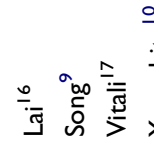 & 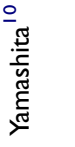 & 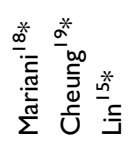 \\
\hline
\end{tabular}

LH and RFA for the treatment of hepatocellular carcinoma. The characteristics of the ten included retrospective studies are shown in Table 1. The baseline characteristics of the patients involved in each study are shown in Table 2.

A study by Casaccia et al was published in 2015 and was updated in 2017, and the perioperative and survival data were extracted from both studies. ${ }^{8,12}$ A study by Harada, Ito, and Lin performed propensity-score matching (PSM) analysis. ${ }^{13-15}$ To guarantee a sufficient sample size (the use of PSM always leads to a decreased sample size), the data before the PSM was performed were used in our study, and the data after the PSM was performed were used to confirm our results by using it to replace the data from before the PSM.

\section{Overall survival}

The 1-, 3-, and 5-year overall survival rates were compared in 8 studies (Figure 2). ${ }^{8-10,13,15-17}$ There were significantly better overall survival rates for the patients who underwent LH than the patients who underwent RFA in terms of all three overall survival rates. For 1-year overall survival, 6 of the 7 included studies reported a similar survival rate for RFA and for LH. Only the study by Lin et al, which had a relatively large sample size, reported a significantly better 1-year overall survival rate for $\mathrm{LH}$ than for RFA. Additionally, the sensitivity analysis that was performed by removing the study by Lin et al showed that this study significantly affected the results. The same situation was also observed for the comparison of the 3 -year overall survival rate. However, the meta-analysis showed that there was a significantly better 5 -year overall survival rate in the LH group than in the RFA group $(\mathrm{OR}=0.53,95 \% \mathrm{CI}=0.40,0.69, p<0.001)$, and this result was relatively stable based on the sensitivity analysis test. The funnel plot for the comparison of overall survival detected no publication bias (Figure S1).

\section{Disease-free survival}

The 1- and 3-year disease-free survival rates were compared in 6 studies, and the 5-year disease-free survival rate was compared in 5 studies (Figure 3). ${ }^{8-10,13,15,18}$ There was a significantly better disease-free survival rate for LH than for RFA in terms of the 1- and 3-year diseasefree survival rates. For the 5-year disease-free survival rate, the meta-analysis showed a comparable disease-free rate for RFA and for $\mathrm{LH}(\mathrm{OR}=0.77,95 \% \mathrm{CI}=0.55,1.08$, $p=0.130$ ). The sensitivity analysis showed that the results 


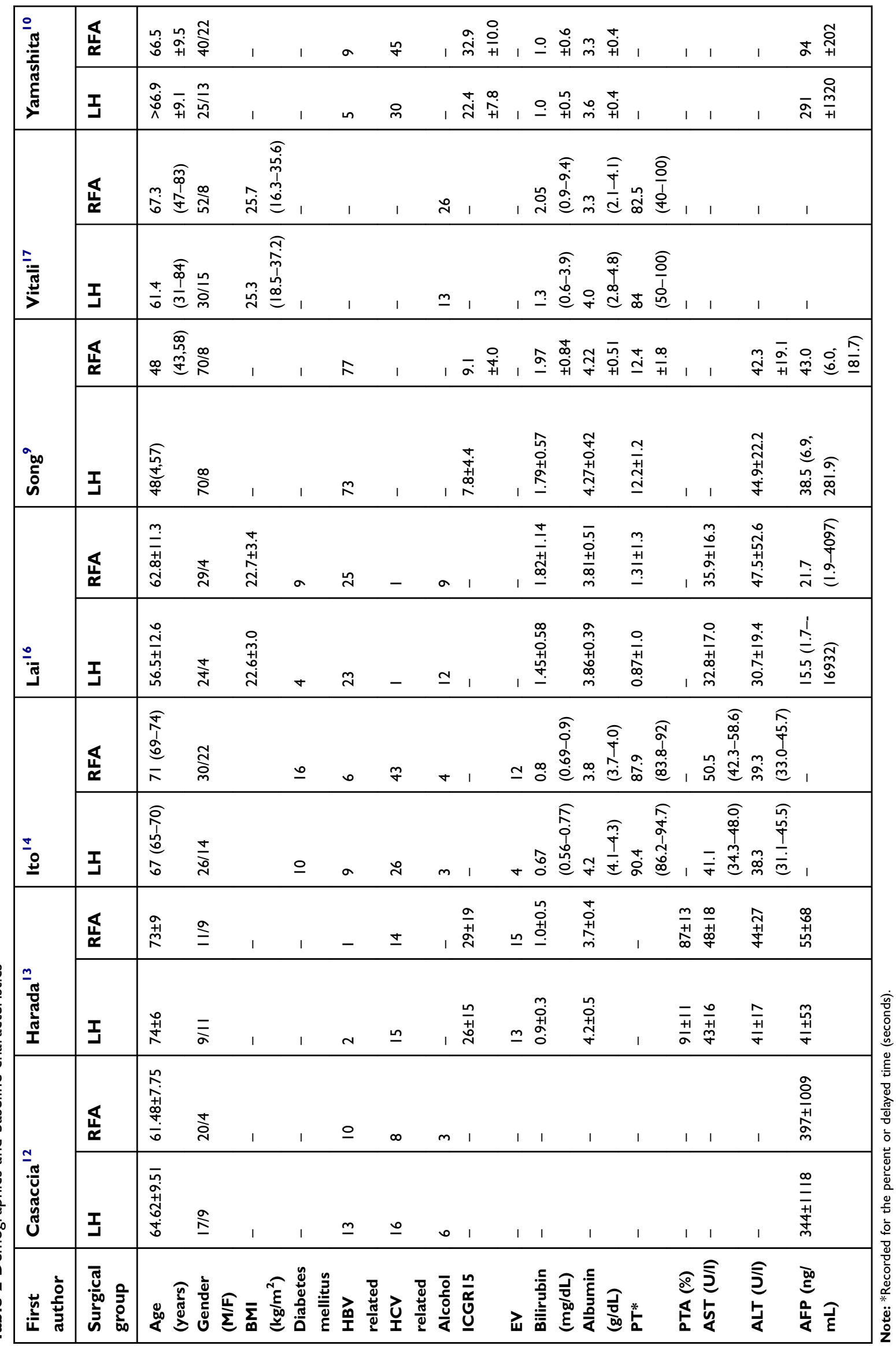




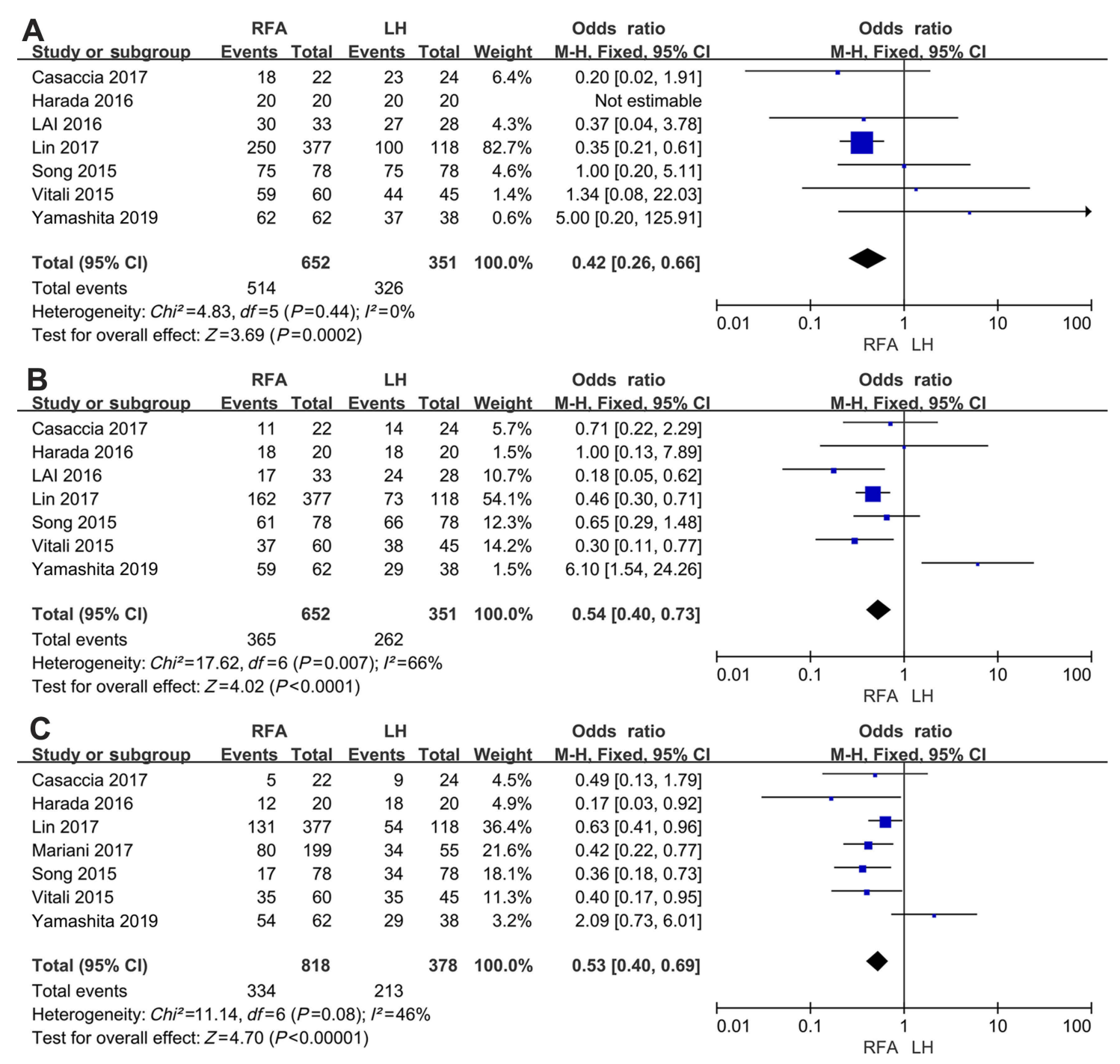

Figure 2 The forest plot of the comparison in terms of (A) I-year, (B) 3-year rate, and (C) 5-year overall survival rate.

of the above three comparisons were stable, and the funnel plot detected no publication bias (Figure S2).

\section{Local recurrence rate}

Local recurrence was defined as intrahepatic recurrence, including recurrence at the site of resection or of the ablated tumor. Only 2 studies were used for the comparison of the 1-, 2-, and 3-year local recurrence rates. ${ }^{14,16}$ There was a significantly lower local recurrence rate for LH than for RFA in terms of the 1- to 3-year local recurrence rates (Figure 4). The sensitivity analysis confirmed the stability of these results, and the assessment of the risk of bias was not performed because only two studies were included in this analysis.

\section{Complications}

The surgery-related complications were assessed in 10 studies (Figure 5). ${ }^{9,10,12-19}$ The present meta-analysis found that the RFA group had a significantly lower complication rate compared with that of the LH group $(\mathrm{OR}=0.64,95 \% \mathrm{CI}=0.46,0.89, p=0.008)$. Then, the sensitivity analysis confirmed the stability of the results, and the funnel plot detected no publication bias (Figure S3).

\section{The subgroup analysis in studies of small hepatocellular carcinomas}

We performed a subgroup analysis of the studies of small hepatocellular carcinomas. The definitions of "small hepatocellular carcinomas" were not completely consistent (Table 1). 


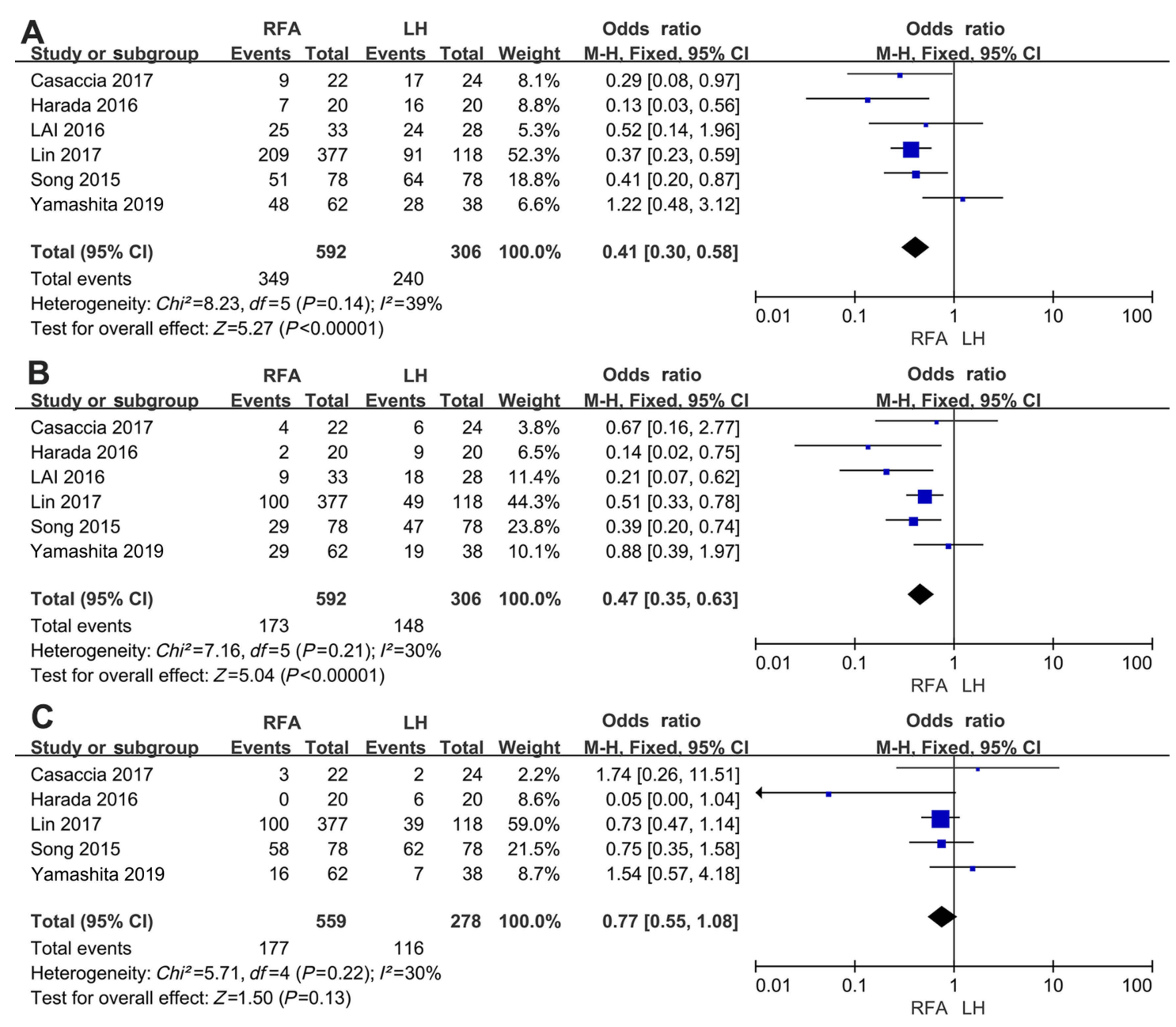

Figure 3 The forest plot of the comparison in terms of (A) I-year, (B) 3-year rate, and (C) 5-year disease-free survival rate.

There were 8 small hepatocellular carcinoma-related studies, and 7 of these studies defined "small hepatocellular carcinomas" according to the Barcelona Clinic Liver Cancer staging system (stage 0 or A) and the Milan criteria. ${ }^{8-10,13,14,18-17}$ The results of the subgroup analysis showed that there was no significant difference between the LH and RFA groups in terms of the 1- and 3-year overall survival rates (Figure 6). However, the LH group had a significantly better 5-year overall survival rate than the RFA group $(\mathrm{OR}=0.47,95 \% \mathrm{CI}=0.33$, $0.66, p=p<0.001$ ). Regarding the disease-free survival rate, there was no significant difference between the LH and RFA groups in terms of the 5-year disease-free survival rate $(\mathrm{OR}=0.83,95 \% \mathrm{CI}=0.49,1.40, p=0.480)$. In contrast, the $\mathrm{LH}$ group had a significantly better 1 - and 3 -year disease survival rate than the RFA group (Figure 7). There was still a lower complication rate in the RFA group than in the LH group (Figure S4). The assessment of the risk of bias in the subgroup analysis was not performed because fewer studies were included.

\section{Discussion}

The present meta-analysis revealed that $\mathrm{LH}$ was superior to RFA in terms of the 5-year overall survival rate. Although the 1- and 3-year overall survival rates were also better in the $\mathrm{LH}$ group than in the RFA group, the results were not stable based on the sensitivity analysis. The subgroup analysis of the small HCCs confirmed that there was an improved 5-year overall survival rate for the LH group than for the RFA group, and no significant difference in the 1- and 3-year overall survival rates was detected. Additionally, a lower local recurrence rate but a higher complication rate was detected in the LH group compared with that of the RFA group.

Open hepatectomy $(\mathrm{OH})$ is a traditional and wellestablished method for the curative treatment of HCCs. ${ }^{20}$ 


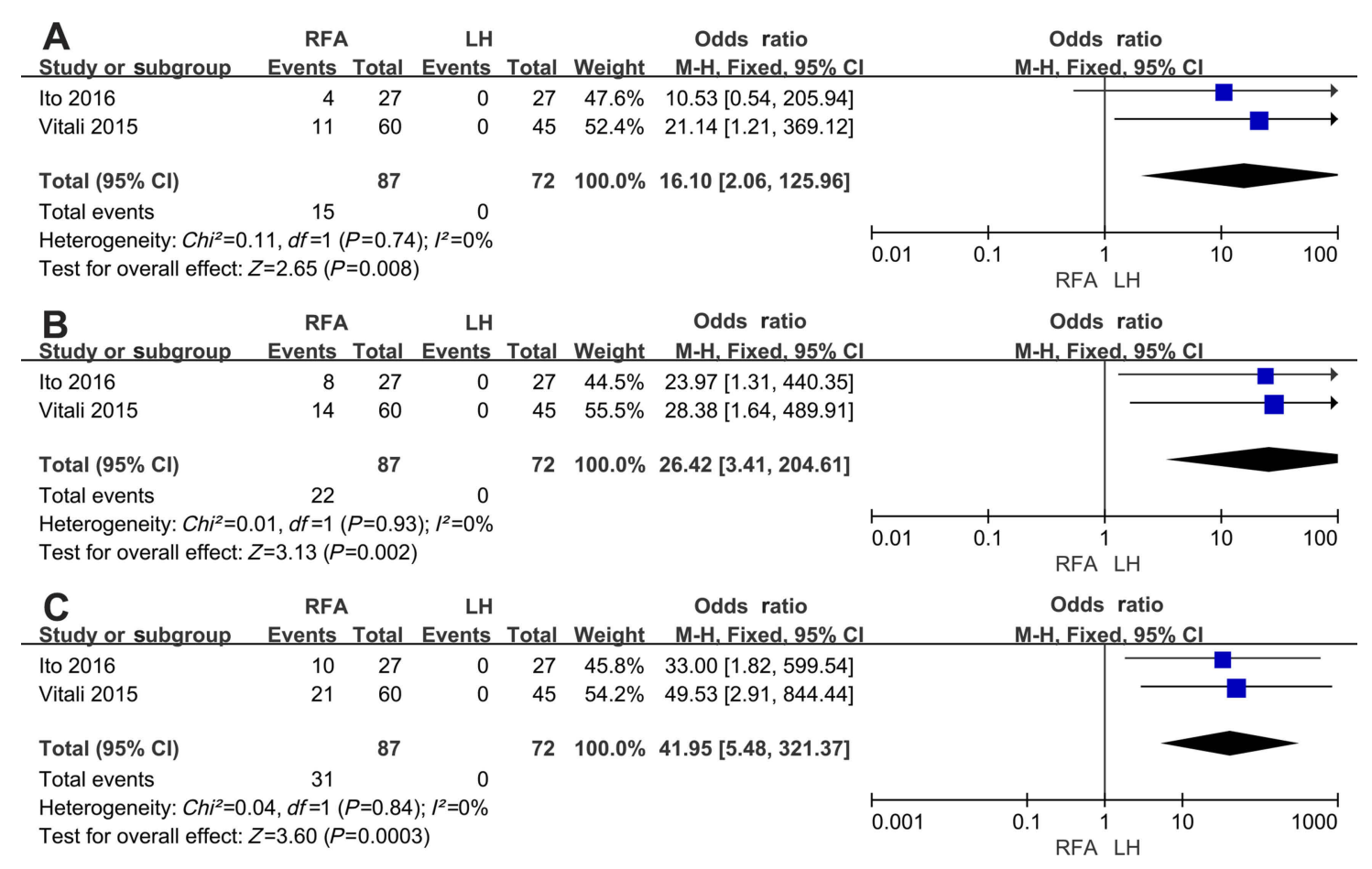

Figure 4 The forest plot of the comparison in terms of (A) I-year, (B) 2-year rate, and (C) 3-year local recurrence rate.

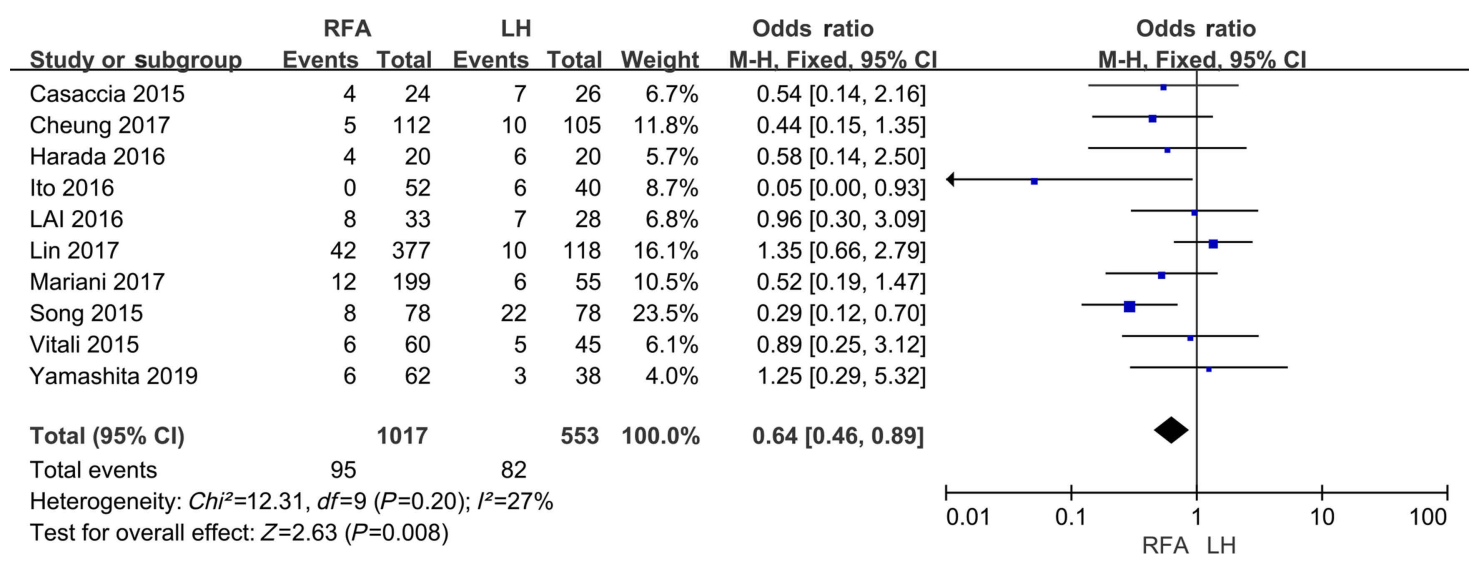

Figure $\mathbf{5}$ The forest plot of the comparison in terms of the complications.

Nevertheless, the large incision, wide extent of resection, and the relatively large amount of blood loss of open surgery always lead to more trauma for patients. $^{21}$ Therefore, $\mathrm{OH}$ was considered suitable for patients with normal liver function but not for patients with severe cirrhosis or poor functional reserve. For these reasons, LH emerged as a viable alternative to open surgery, while LH has the characteristics of a minimally invasive approach. A recent pooled analysis showed that a lower incidence of postoperative ascites and liver failure was observed in the LH group compared with that in the $\mathrm{OH}$ group, and the oncological results did not show any significant differences. ${ }^{22}$ The superiority of LH compared to $\mathrm{OH}$ among selected cirrhotic patients extended the indications for LH to patients with severe cirrhosis and HCCs.

RFA, which is a commonly used minimally invasive treatment, has been widely used for HCCs. Several randomized controlled trials (RCTs) and meta-analyses have compared the efficacy of RFA and $\mathrm{OH}^{23-25}$ Radiofrequency ablation was shown to be an effective treatment for early stage HCCs, with a comparable prognostic outcome and a lower complication rate than open hepatectomy. Therefore, surgeons have gradually started to pay attention to the comparisons of these two 


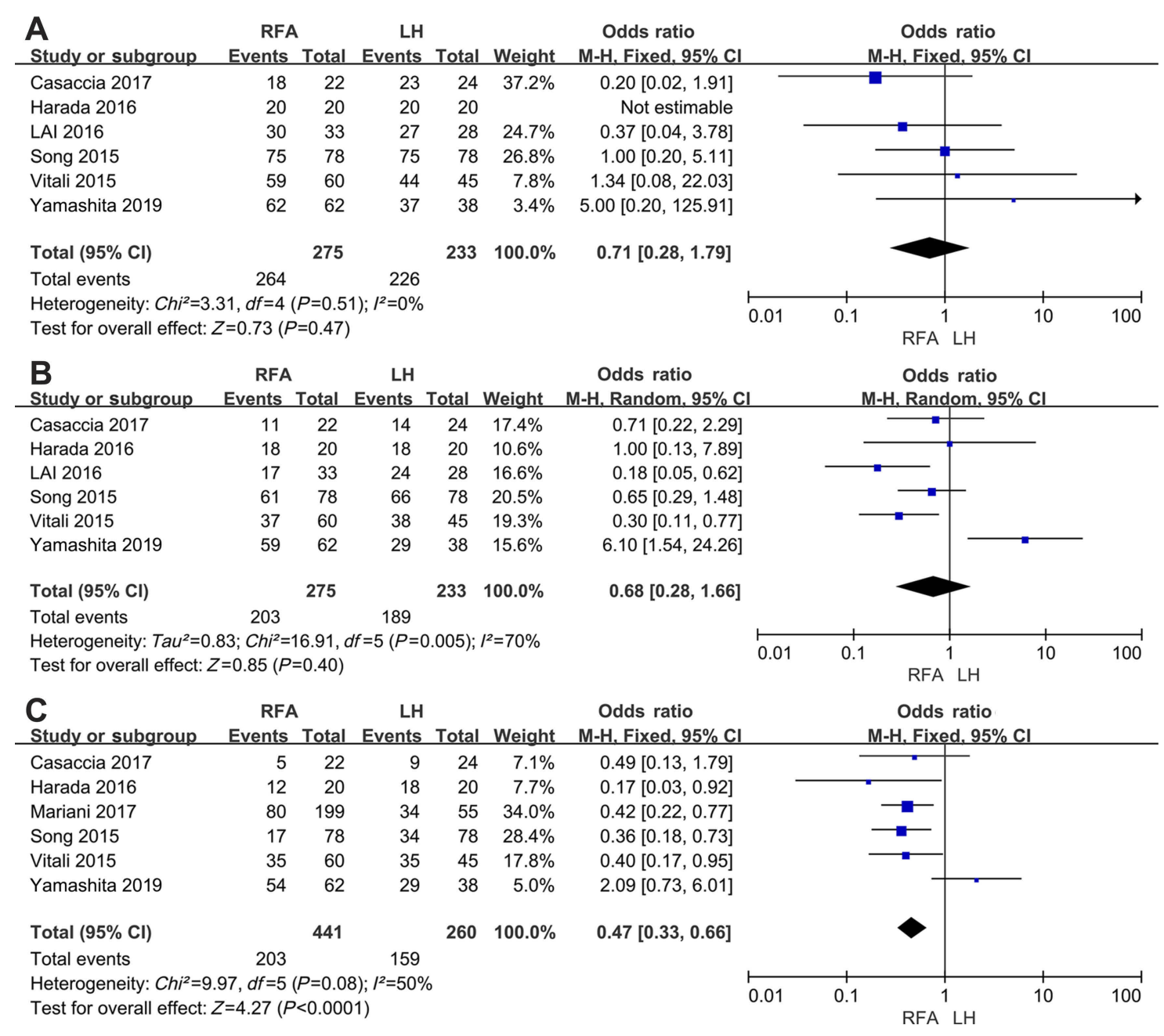

Figure 6 The forest plot of the comparison in terms of (A) I-year, (B) 3-year rate, and (C) 5-year overall survival rate (subgroup analysis for small hepatocellular carcinoma).

minimally invasive methods for the curative treatment of HCCs in recent years.

There are still no published RCTs and meta-analyses comparing LH and RFA in HCCs. One double-blind RCT (NCT02243384) and another randomized clinical trial (NCT02535117) are both recruiting patients. Our electronic search yielded 10 retrospective studies, and the pooling results showed that the LH group had a better long-term prognosis and a lower recurrence rate but a higher complication rate compared to those of the RFA group. The higher recurrence rate in the RFA group might be one of the explanations of the worse long-term prognosis, and the recurrence rate of the RFA in our study was persistent with the previous studies. ${ }^{16}$ The microscopic tumor foci that have not been detected by imaging or malignant cells that spread during radiofrequency ablation were believed to be the main reasons of recurrence in the RFA. $^{26,27}$ Although the LH was evaluated to be minimal invasive, less bleeding, shorter length of stay, and recent evidences showed that the LH had a significantly less complication rate compared with the $\mathrm{OH}$. The pooling results in our study found out that the gap in terms of complication rate between the hepatectomy and RFA was still not closed.

In the study by Yamashita et al, a multimodal strategy, in which the surgeons selected a strategy from among percutaneous RFA, laparoscopic RFA, thoracoscopic RFA, or a combination according to the locations of tumors, was used. ${ }^{10}$ The RFA group was shown to have a similar overall and disease-free survival rate and a shorter hospital stay compared with those of the LH group in this study. However, the multimodal strategy was only used in the RFA group and not in the LH group. Therefore, we do not know whether the results would change if both the RFA and LH groups had used the multimodal strategy. We were not able to perform subgroup analysis based on the location of the tumors or on the surgical approaches in the present meta-analysis. 


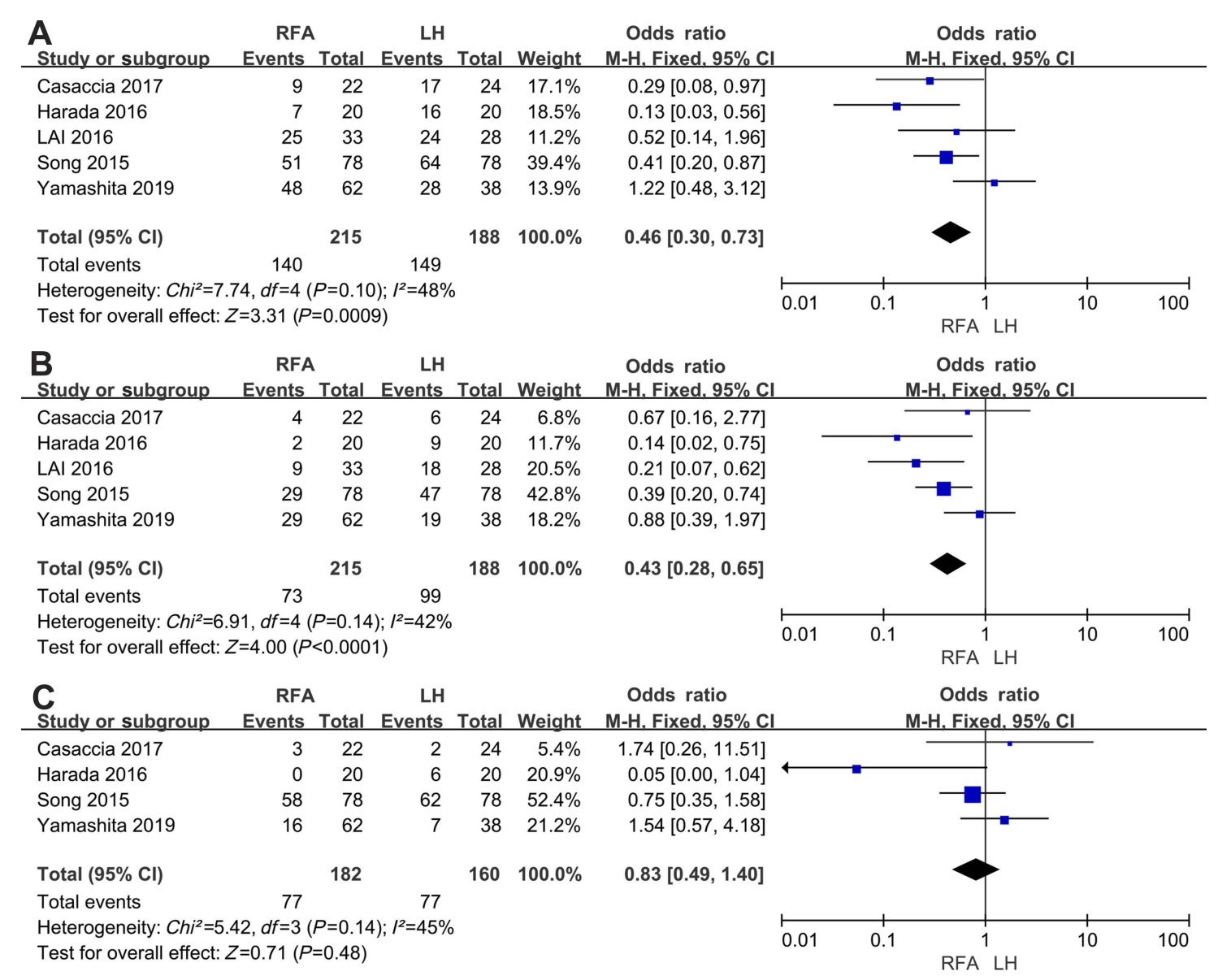

Figure 7 The forest plot of the comparison in terms of (A) I-year, (B) 3-year rate, and (C) 5-year disease-free survival rate (subgroup analysis for small hepatocellular carcinoma).

There are several limitations of this meta-analysis that should be taken into consideration when interpreting our results. First, all the 10 included studies were retrospective studies, and some of them were conference abstract which could not be evaluated by quality assessment. There are almost certainly election biases based on the retrospective nature of studies included (technical or patient or liver or tumor characteristics) that led to one technique being chosen over the other that might bias results (even with propensity matching). Second, the heterogeneity of several results was slightly high. Third, there was heterogeneity in the laparoscopic operation materials in the LH group and the various power selections and durations of the ablation and the definition of complete necrosis in the RFA group, and the strategy for the treatment of recurrence. Fourth, publication bias may have influenced the authenticity of our results.

In summary, the present meta-analysis revealed that the LH led to a better 5-year overall survival rate and a lower recurrence rate but a higher complication rate compared with those of RFA. However, our results could not provide conclusive evidence for the superiority of LH over RFA for the treatment of HCCs due to the inclusion of retrospective studies. Additional high-quality RCTs that investigate the long-term efficacy of LH and RFA are needed to provide more reliable evidence. The multimodal strategy that comprised different surgical approaches should be considered for tumors from different locations in future studies.

\section{Acknowledgments}

We are especially thankful to $\mathrm{Dr}$ Wei $\mathrm{Hu}$ from the Hangzhou First People's Hospital for his help on the critical revise of the manuscript.

\section{Disclosure}

The authors report no conflicts of interest in this work. 


\section{References}

1. Ferlay J, Soerjomataram I, Dikshit R, et al. Cancer incidence and mortality worldwide: sources, methods and major patterns in GLOBOCAN 2012. Int $J$ Cancer. 2015;136(5):E359-E386. doi:10.1002/ijc. 29210

2. Omata M, Cheng AL, Kokudo N, et al. Asia-Pacific clinical practice guidelines on the management of hepatocellular carcinoma: a 2017 update. Hepatol Int. 2017;11(4):317-370. doi:10.1007/s12072-0179799-9

3. Leoni S, Piscaglia F, Serio I, et al. Adherence to AASLD guidelines for the treatment of hepatocellular carcinoma in clinical practice: experience of the bologna liver oncology group. Dig Liver Dis. 2014;46(6):549-555. doi:10.1016/j.dld.2014.02.012

4. Lai Q, Vitale A, Rossi M, Cillo U, Lerut J. Which is the true role of bridging therapies for $\mathrm{HCC}$ patients waiting for liver transplantation? Ann Surg. 2018;268(6):e56-e57. doi:10.1097/SLA.0000000000002575

5. Yamashita Y, Tsuijita E, Takeishi K, et al. Trends in surgical results of hepatic resection for hepatocellular carcinoma: 1,000 consecutive cases over 20 years in a single institution. Am J Surg. 2014;207 (6):890-896. doi:10.1016/j.amjsurg.2013.07.028

6. Forner A, Reig ME, de Lope CR, Bruix J. Current strategy for staging and treatment: the BCLC update and future prospects. Semin Liver Dis. 2010;30(1):61-74. doi:10.1055/s-0030-1247133

7. Jiang B, Yan XF, Zhang JH. Meta-analysis of laparoscopic versus open liver resection for hepatocellular carcinoma. Hepatol Res. 2018;48(8):635-663. doi:10.1111/hepr.13061

8. Casaccia M, Santori G, Bottino G, Diviacco P, Andorno E. Laparoscopic resection vs laparoscopic radiofrequency ablation for the treatment of small hepatocellular carcinomas: A single-center analysis. World J Gastroenterol. 2017;23(4):653-660. doi:10.3748/wjg.v23.i4.653

9. Song J, Wang Y, Ma K, et al. Laparoscopic hepatectomy versus radiofrequency ablation for minimally invasive treatment of single, small hepatocellular carcinomas. Surg Endosc. 2016;30 (10):4249-4257. doi:10.1007/s00464-015-4737-1

10. Yamashita YI, Imai K, Kaida T, et al. Multimodal radiofrequency ablation versus laparoscopic hepatic resection for the treatment of primary hepatocellular carcinoma within Milan criteria in severely cirrhotic patients: long-term favorable outcomes over 10 years. Surg Endosc. 2019;33(1):46-51. doi:10.1007/s00464-018-6264-3

11. Lin H, Jin Z, Zhu Y, Diao M, Hu W. Anal fistula plug vs rectal advancement flap for the treatment of complecryptoglandular anal fistulas: a system review and meta-analysis of studies with long-term follow-up. Colorectal Dis. 2018.

12. Casaccia M, Santori G, Bottino G, et al. The procedure outcome of laparoscopic resection for 'small' hepatocellular carcinoma is comparable to vlaparoscopic radiofrequency ablation. $J$ Minim Access Surg. 2015;11(4):231-235. doi:10.4103/0972-9941.144093

13. Harada N, Maeda T, Yoshizumi T, et al. Laparoscopic liver resection is a feasible treatment for patients with hepatocellular carcinoma and portal hypertension. Anticancer Res. 2016;36(7):3489-3497.

14. Ito $T$, Tanaka $S$, Iwai $S$, et al. Outcomes of laparoscopic hepatic resection versus percutaneous radiofrequency ablation for hepatocellular carcinoma located at the liver surface: a case-control study with propensity score matching. Hepatol Res. 2016;46(6):565-574. doi:10.1111/hepr.12592
15. Lin M, Peng Z, Chen S, Dai Z, Wei G, Kuang M. Laparoscopic surgery versus radiofrequency ablation for the treatment of recurrent hepatocellular carcinoma: a propensity score matching study. Hepatol Int. 2017;11(1):S67.

16. Vitali GC, Laurent A, Terraz S, et al. Minimally invasive surgery versus percutaneous radio frequency ablation for the treatment of single small $(</=3 \mathrm{~cm})$ hepatocellular carcinoma: a case-control study. Surg Endosc. 2016;30(6):2301-2307. doi:10.1007/s00464015-4295-6

17. Mariani NM, Santambrogio R, Barabino M, Scifo G, Giovenzana M, Opocher E. Laparoscopic liver resection vs radiofrequency ablation in cirrhotic patients with hepatocellular carcinoma: towards the 'gold standard'. Surg Endosc Other Interventional Tech. 2017;31(2):S33. doi:10.1007/s00464-016-5054-z

18. Lai C, Jin RA, Liang X, Cai XJ. Comparison of laparoscopic hepatectomy, percutaneous radiofrequency ablation and open hepatectomy in the treatment of small hepatocellular carcinoma. J Zhejiang Univ Sci B. 2016;17(3):236-246. doi:10.1631/jzus. B1500322

19. Cheung TT, Chan ACY, Chok KSH, Lo CM. Laparoscopic liver resection versus percutaneous radiofrequency ablation in patients with hepatocellular carcinoma and liver cirrhosis: a case-matched study. Lancet. 2017;390(SPEC.ISS1):39. doi:10.1016/S0140-6736 (17)33177-X

20. Bruix J, Sherman M. Management of hepatocellular carcinoma: an update. Hepatology (Baltimore, Md). 2011;53(3):1020-1022. doi:10.1002/hep.24199

21. Benvegnu L, Gios M, Boccato S, Alberti A. Natural history of compensated viral cirrhosis: a prospective study on the incidence and hierarchy of major complications. Gut. 2004;53(5):744-749. doi:10.1136/gut.2003.020263

22. Morise Z, Ciria R, Cherqui D, Chen KH, Belli G, Wakabayashi G. Can we expand the indications for laparoscopic liver resection? A systematic review and meta-analysis of laparoscopic liver resection for patients with hepatocellular carcinoma and chronic liver disease. J Hepatobiliary Pancreat Sci. 2015;22(5):342-352. doi:10.1002/ jhbp. 215

23. Xu XL, Liu XD, Liang M, Luo BM. Radiofrequency ablation versus hepatic resection for small hepatocellular carcinoma: systematic review of randomized controlled trials with meta-analysis and trial sequential analysis. Radiology. 2018;287(2):461-472. doi:10.1148/ radiol.2017162756

24. Chen MS, Li JQ, Zheng Y, et al. A prospective randomized trial comparing percutaneous local ablative therapy and partial hepatectomy for small hepatocellular carcinoma. Ann Surg. 2006;243 (3):321-328. doi:10.1097/01.sla.0000201480.65519.b8

25. Feng K, Yan J, Li X, et al. A randomized controlled trial of radiofrequency ablation and surgical resection in the treatment of small hepatocellular carcinoma. $J$ Hepatol. 2012;57(4):794-802. doi:10.1016/j.jhep.2012.05.007

26. Weis S, Franke A, Mossner J, Jakobsen JC, Schoppmeyer K. Radiofrequency (thermal) ablation versus no intervention or other interventions for hepatocellular carcinoma. Cochrane Database Syst Rev. 2013;(12):Cd003046.

27. Afaneh C, Kluger MD. Laparoscopic liver resection: lessons at the end of the second decade. Semin Liver Dis. 2013;33(3):226-235. doi:10.1055/s-0033-1351780 


\section{Supplementary materials}

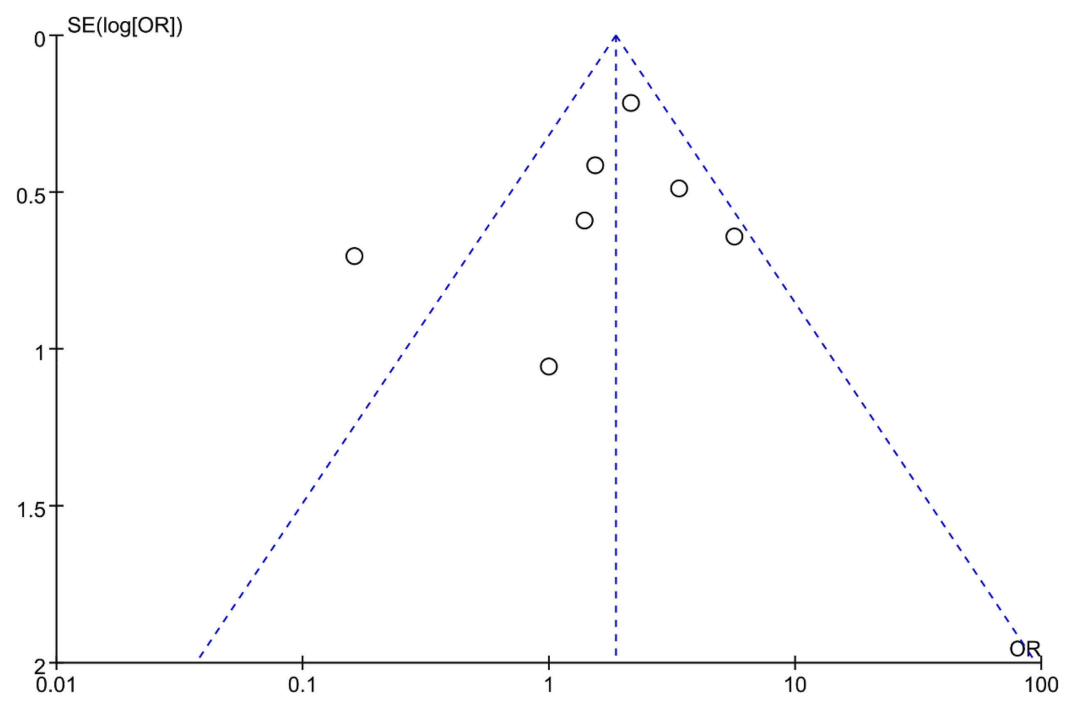

Figure SI The funnel plot for the comparison of overall survival rate.

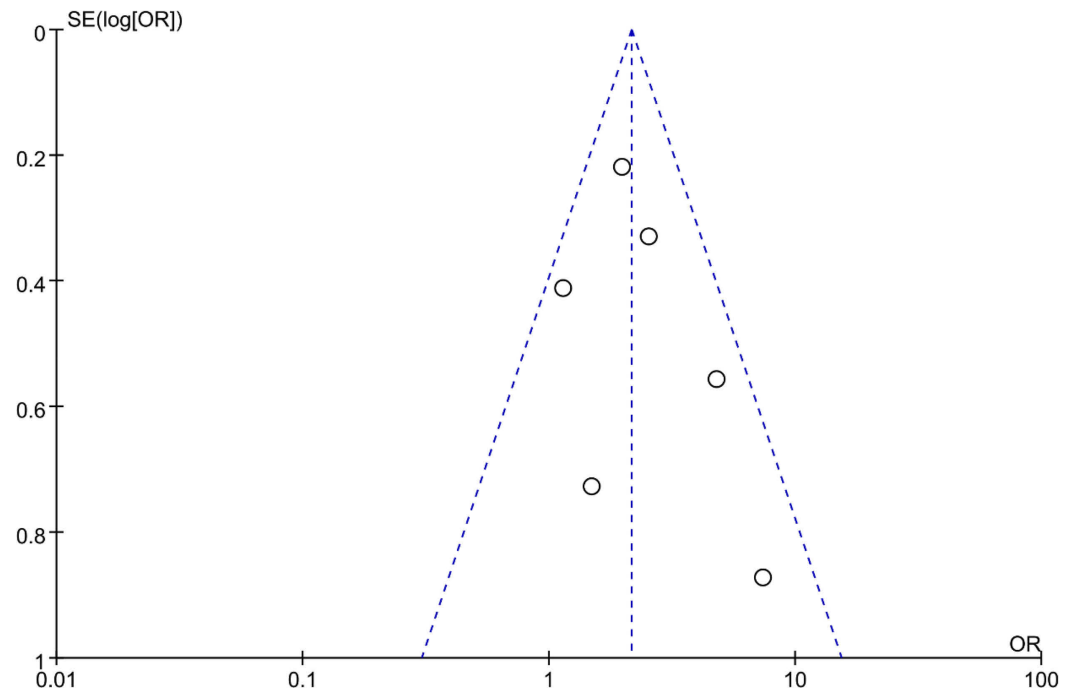

Figure S2 The funnel plot for the comparison of disease-free survival rate. 


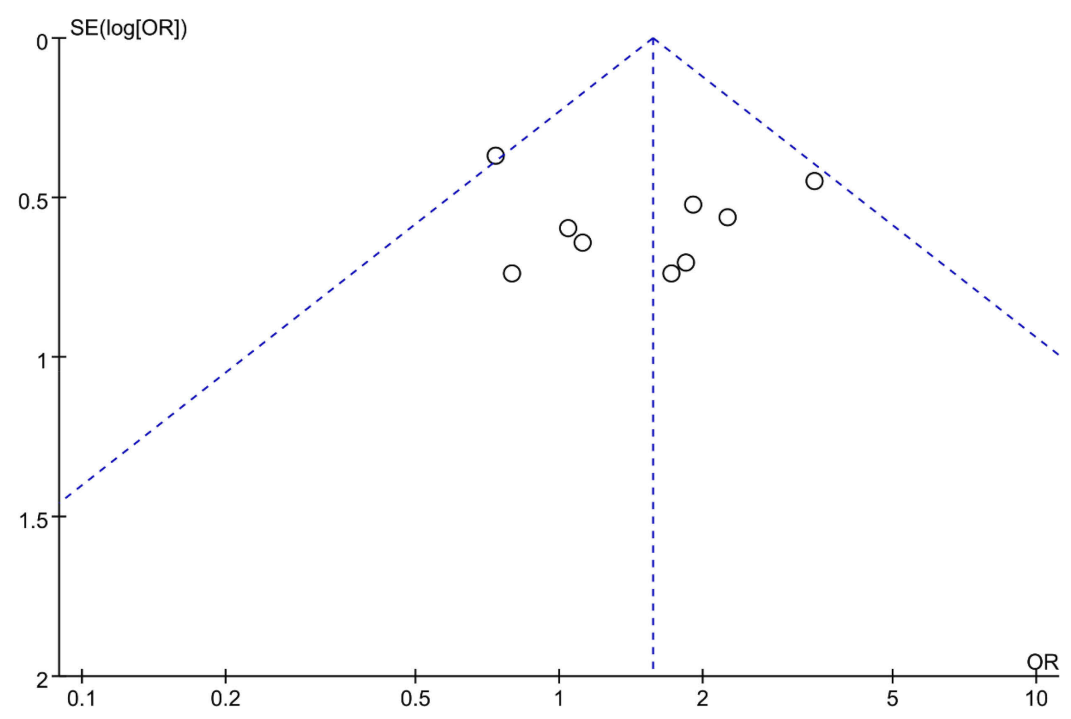

Figure S3 The funnel plot for the comparison of complications.

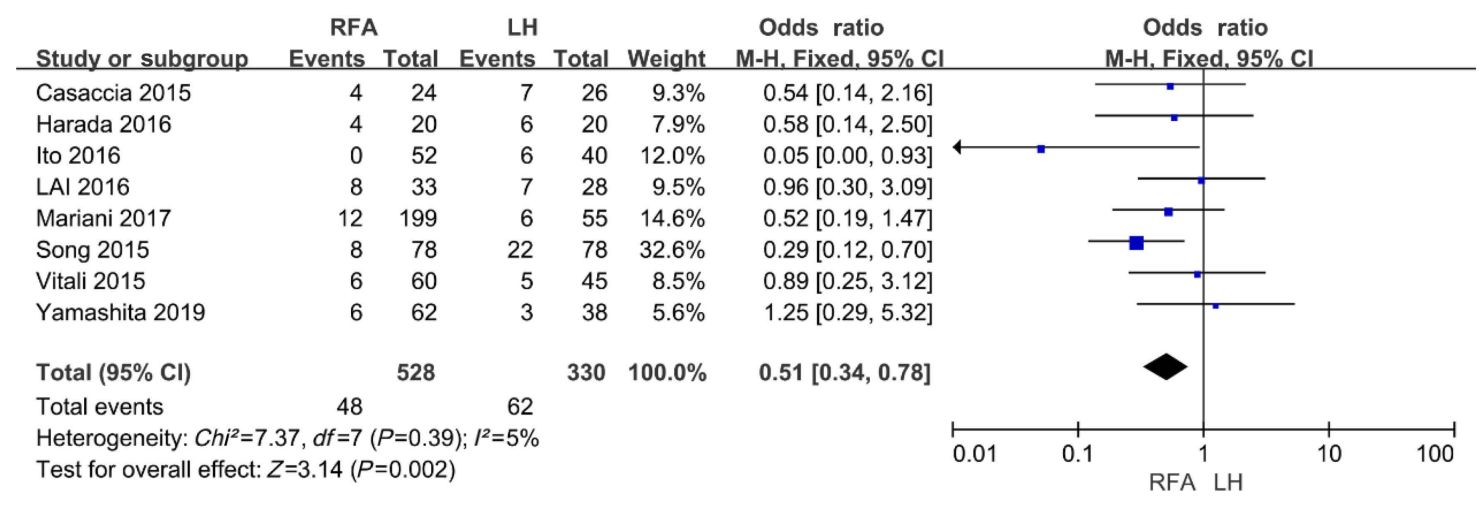

Figure S4 Forest plots of studies included comparing the complications between LH and RFA (subgroup analysis for small hepatocellular carcinoma).

\section{References}

1. Casaccia M, Santori G, Bottino G, et al. The procedure outcome of laparoscopic resection for 'small' hepatocellular carcinoma is comparable to vlaparoscopic radiofrequency ablation. J Minim Access Surg. 2015;11(4):231-235. doi:10.4103/0972-9941.144093

2. Harada N, Maeda T, Yoshizumi T, et al. Laparoscopic liver resection is a feasible treatment for patients with hepatocellular carcinoma and portal hypertension. Anticancer Res. 2016;36(7):3489-3497.

3. Ito T, Tanaka S, Iwai S, et al. Outcomes of laparoscopic hepatic resection versus percutaneous radiofrequency ablation for hepatocellular carcinoma located at the liver surface: a case-control study with propensity score matching. Hepatol Res. 2016;46(6):565-574. doi:10.1111/hepr.12592

4. Lai C, Jin RA, Liang X, Cai XJ. Comparison of laparoscopic hepatectomy, percutaneous radiofrequency ablation and open hepatectomy in the treatment of small hepatocellular carcinoma. J Zhejiang Univ Sci B. 2016;17(3):236-246. doi:10.1631/jzus.B1500322
5. Mariani NM, Santambrogio R, Barabino M, Scifo G, Giovenzana M, Opocher E. Laparoscopic liver resection vs radiofrequency ablation in cirrhotic patients with hepatocellular carcinoma: towards the 'gold standard'. Surg Endosc Other Interventional Tech. 2017;31(2):S33. doi:10.1007/s00464-016-5054-z

6. Song J, Wang Y, Ma K, et al. Laparoscopic hepatectomy versus radiofrequency ablation for minimally invasive treatment of single, small hepatocellular carcinomas. Surg Endosc. 2016;30 (10):4249-4257. doi:10.1007/s00464-015-4737-1

7. Vitali GC, Laurent A, Terraz S, et al. Minimally invasive surgery versus percutaneous radio frequency ablation for the treatment of single small $(</=3 \mathrm{~cm})$ hepatocellular carcinoma: a case-control study. Surg Endosc. 2016;30(6):2301-2307. doi:10.1007/s00464-015-4295-6

8. Yamashita YI, Imai K, Kaida T, et al. Multimodal radiofrequency ablation versus laparoscopic hepatic resection for the treatment of primary hepatocellular carcinoma within Milan criteria in severely 400 cirrhotic patients: long-term favorable outcomes over 10 years. Surg Endosc. 2019;33(1):46-51. doi:10.1007/s00464-018-6264-3 


\section{Publish your work in this journal}

Cancer Management and Research is an international, peer-reviewed The manuscript management system is completely online and includes open access journal focusing on cancer research and the optimal use of a very quick and fair peer-review system, which is all easy to use. preventative and integrated treatment interventions to achieve improved Visit http://www.dovepress.com/testimonials.php to read real quotes outcomes, enhanced survival and quality of life for the cancer patient.

from published authors. 